\title{
Maximum Utilization in Operations Scheduling for Multiple Machines and Batches in Additive Manufacturing
}

\author{
Salvador Castillo-Rivera \\ Institute of Physics of Cantabria, Spanish Council for Scientific Research, University of Cantabria, Santander, Spain \\ E-mail: salvadorcastillorivera@gmail.com
}

Received: 1 May 2021; Revised: 8 July 2021; Accepted: 22 July 2021

\begin{abstract}
Scheduling and sequencing are forms of decision making that present a fundamental role in Additive Manufacturing (AM). This work studies the scheduling for a set of three-dimensional (3D) printers when the maximum utilization is used as an indicator. The aim is to establish a criterion that minimizes the use of available resources. A program has been done by using the software package Python and this has allowed studying the effect that the maximum utilization presents on operation scheduling. As evaluated a large number of pieces of various sizes, the analysis is found to be effective in yielding near-optimal solutions. The obtained outcomes have identified aspects of the production such as production planning and control as well as work organization.
\end{abstract}

Keywords: 3D printer, batches, scheduling, multiple machines

\section{Introduction}

New scheduling models are needed to adapt the traditional scheduling rules for Additive Manufacturing (AM). Furthermore, the manufacturing planning problem is being addressed in terms of minimizing the total cost of production. As a consequence, scheduling for a set of three-dimensional (3D) printers should be studied when the maximum utilization is used as an indicator to determine a criterion that minimising the use of available resources in the AM industry.

According to Weller et al. [1], 3D printing is considered an essential part of the new industrial revolution and Dilberoglu et al. [2] has reported that the fourth industrial revolution notably depends on the capabilities of AM. Pour et al. [3] presented a set of proposals to re-establish the production system as well as the supply chain to allow AM as a feasible and functional system. Demirel et al. [4] have set out that the demand is able to generate frequent changes in production plans, which produces uncertainty in AM companies. As shown, the 3D printing market presents a challenge due to the growing demand that this industry is facing and it should provide a response to the placement problem of different matches as several printers are available. As a consequence of this, AM should face the assignation problem and a methodology should be derived to determine the machine's utilization, along with a set of batches to estimate the maximum utilization per AM machine.

The advances in AM technologies have generated a significant increase in these types of production processes in industries. In order to meet customers' demands and make the investment in these machines profitable, it is required

Copyright (C2021 Salvador Castillo-Rivera

DOI: https://doi.org/10.37256/dmt.112021909

This is an open-access article distributed under a CC BY license

(Creative Commons Attribution 4.0 International License)

https://creativecommons.org/licenses/by/4.0/ 
to study the production organization in AM. Aloui et al. [5] have dealt with the scheduling and nesting problem of production with technological constraints. The aim was to minimize the total delay of the parts to be fabricated and to maximize the use rate of the AM machines. Two models were proposed for powder-based laser technologies and multijet fusion technology, to evaluate the production time in AM based on real data. The nesting and scheduling problem was modelled by mixed linear programming. The numerical outcomes proved that the solutions were close to the lower bounds proposed.

A number of researches have addressed the production planning and scheduling issues in additive manufacturing. Li et al. [6] presented a mathematical model which was coded in CPLEX. Two heuristic procedures were also developed in JavaScript, called 'best-fit' and 'adapted best-fit', respectively. Experimental tests displayed that both heuristics provided encouraging results. Chergui et al. [7] studied the orders received from different distributed customers by due dates. A mathematical formulation of the problem was presented and a heuristic approach was also proposed. Experimental tests were done using the proposed heuristic, underlining the influence of planning/scheduling on the optimized production with AM. Dvorak et al. [8] analysed a set of parts with unique configurations to be printed by a set of machines whereas deadlines were met and production time was minimized. Preliminary experimental results were presented and the main contribution of the work was a new model. It should be extended into an optimization model that captures all the discrete decision making challenges in AM. Fera et al. [9] conducted the research literature on AM application in the industrial sector. The mechanical and chemical features of the components fabricated by $\mathrm{AM}$ and the management topic were studied. On the other hand, a number of cost models of AM have been proposed and investigated, such as the work presented by Fera et al. [10]. A model was developed to assess process costs of AM processes including selective laser sintering, stereolithography and electron beam melting. Costabile et al. [11] studied the existing literature concerning the cost models for AM from an operation management's viewpoint. The strengths and weaknesses of all models were also discussed.

As Stittgen et al. [12] have pointed out AM is on the brink of industrialization and the service providers must configure the production environment in a way that allows large-capacity utilization and short throughput times while the work in process is minimized. They set out the impact of a service provider's facility configuration regarding machine pool, operator availability and distribution of work content on the production system's utilization. The validation of the model was based on empirical data gathered on the shop floor of GKN Additive, a global AM service provider. Due to the new requirements of AM technology, Fera et al. [13] have presented a new meta-heuristic algorithm based on the Tabu-search algorithms. Czerniachowska et al. [14] have proposed an approach that has covered planning allowances rules to compromise the manufacturing requirements and production cost minimization.

Taking the previous considerations into account, this work tackles operations scheduling in AM, focusing the attention on assigning jobs to machines for the maximum utilization per build platform. A program is done by using the free software packages Python. As a result, the effect of an increase in utilization on determining which machines are on operation scheduling is studied. The aim is to maximize the cost of production for this purpose, the maximum utilization of the printers is considered as an indicator that will enable to characterize the aspects of the production such as AM machines, production planning and control as well as work organization.

In view of these considerations, the main contributions of this article can be summarized as follows: (a) to present a program that provides a solution to the assignation of problem in AM for multiple machines; (b) to figure out the maximum number of parts that a set of 3D printers are able to print when the parts are clustered in batches; (c) to establish operation scheduling from the maximum utilization criterion.

\section{Theoretical framework}

Efficient packing of multiple parts in the 3D printing build platforms can reduce the makespan and the unit cost in AM. Overall, the procedure of AM depends on bodies are packed effectively. Packing algorithms have been studied; Araújo et al. [15] provided a classification of the packing problems in AM and a summary of the benchmarks for the analysis of analogous problems. More realistic future characteristics were also discussed, keeping in mind the promoting research toward efficient and effective $3 \mathrm{D}$ printing.

The packing problems are related to finding a good arrangement of multiple items on different surfaces or regions. 
The process aims to minimize the space between parts, Lodi et al. [16], Renner et al. [17]. The industries involved with mass-production find particular interest in this type of layout, due to a considerable reduction of the production costs. The method of resolution and the geometry of the objects establish the complexity of the problem, Vaissier et al. [18]. The simulation techniques have been demonstrated to be useful, predominantly in the manufacturing domain to ease the estimation of specific parts and products, Takakuwa [19], Castillo-Rivera et al. [20]. Herein, it is figured out the maximum number of pieces per batch that each AM machine can print a program is implemented to derive the possible solutions, see Figure 1.

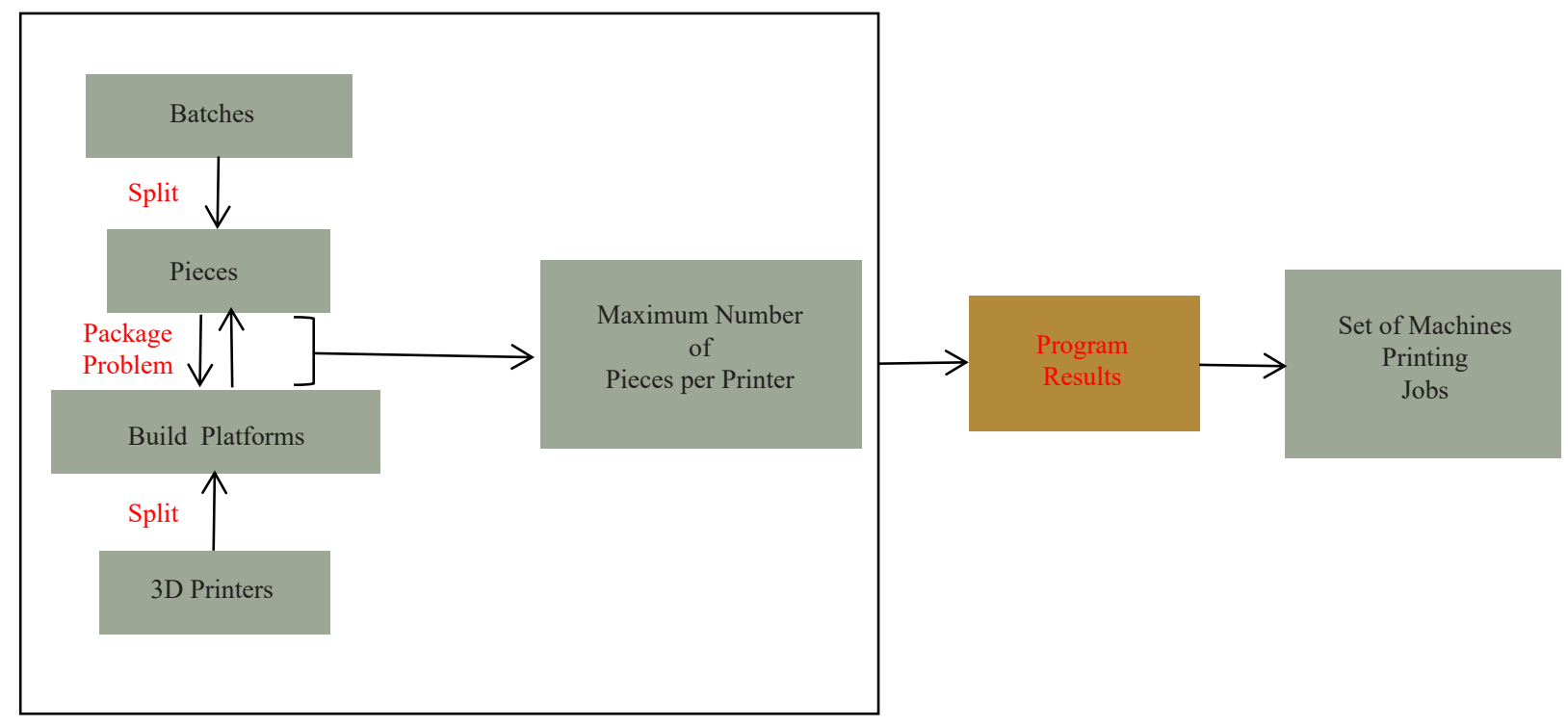

Figure 1. Functional block diagram of the problem study

During production scheduling, machine maintenance has often been ignored. Every machine needs maintenance, which affects the machine's availability and the production schedule. Chung et al. [21] proposed a modified genetic algorithm approach to tackle those distributed scheduling models with maintenance consideration, for minimizing the makespan of the jobs.

Fused Deposition Modeling (FDM) is an AM process often for modelling, prototyping and production applications, Mohamed et al. [22]. According to Dey et al. [23], FDM is used to produce parts and geometrically complex shaped prototypes. It is acquiring popularity because it diminishes cycle time for product development without the requirement of expensive tools. Popescu et al. [24] have indicated that one of the main purposes of FDM is the designing and manufacturing of functional parts for fields such as medicine and engineering.

In this FDM to implement the digital modelling, the first step is to build up a 3D model depending on the request of the customer. This task can be performed through a software package called CAD (Computer-Aided Design). A file should be generated in a suitable format, it is usually taken as Standard Triangle Language (STL) and the whole geometrical information should be included to set up the digital model, this is called the exportation. The digital model must be converted into a list of 3D print out commands in the sliced process, this step is required to execute the corresponding code (g-code), and the connection allows to provide a list of the printer instructions through the USB port or copying the file in a memory card, which could be directly read by the printer. Once this step is done, the printing must be done, finally, the object/objects should be removed from the printing platform.

\section{Problem description}

It is studied how to minimize the cost of production and for this purpose, a basic parameter as the maximum 
machine utilization is used to tackle the reduction of the resources available. For example, a production line can be simulated to study the effect that the maximum utilization of the AM machines on the operations schedule.

There are ten FDM AM machines that have been used for experimentation; these are utilized to simulate a set of commercial 3D printing machines (see Table 1). The configuration will allow the establishment of different production scenarios. The settings present the whole production to be scheduled in an AM plant. A production planning solution is sought to optimize the manufacturing volume for each printer giving suitable values for maximum utilization. It will be seeking satisfying solutions that consider the reduction of production cost and it will be done by maximizing the production volume at each $3 \mathrm{D}$ printing machine.

As a consequence, sets of parts are clustered in batches that should be printed on an ensemble of 3D printers each one of them with a different build platform. The batches will be assigned on a machine by machine basis using the maximum occupation criterion, taking into account the production area and the parts' surfaces.

Table 1. AM machines and their build platforms

\begin{tabular}{cc}
\hline Printer & Build Platform Surface $\left(\mathrm{mm}^{2}\right)$ \\
\hline P1 & $150 \times 85$ \\
P2 & $130 \times 130$ \\
P3 & $160 \times 160$ \\
P4 & $200 \times 200$ \\
P5 & $330 \times 180$ \\
P6 & $300 \times 300$ \\
P7 & $400 \times 250$ \\
P8 & $345 \times 360$ \\
P9 & $750 \times 750$ \\
P10 & $1005 \times 1005$ \\
\hline
\end{tabular}

The first step is to cluster the set of parts (batches) and allocate them to print jobs. Each one of them is composed of a single set of pieces with the same surfaces, this is because the purpose of this work is not to study batches made up of pieces with different dimensions. The following step is how to optimally place these parts into the specified build space (maximum production area). The maximum supported height is neglected because it is linked with the mass of the part; in this work is not a fundamental parameter to reach the main outcomes fixed by the author.

Once the jobs are formed, the objective is to schedule them on a set of AM machines. These have different dimensions for the maximum production area and it is also established that all batches are available at time zero and ready to be processed on the machines. Each machine can process only one job at a time. Thereby, a batch could be processed on different printers at the same time. The scheduling problem is related to finding a solution to assign all batches to the printers, so that maximizes the utility of the machines according to their build surface platform and the corresponding printing job surfaces. The processing time of a batch is affected by the surface area of the parts and build platform surface. Machine utilization is defined by one indicator, the maximum compactness defined as the maximum coverage of the build area.

The following examples show how different combinations of parts affect the operations scheduling: it can be considered a hundred parts with equal heights, production areas and volumes as presented in Table 2. These parts can be scheduled on the AM machines, and therefore, the parts are clustered into the required jobs to satisfy the maximum utility of the machines. 
Table 2. Parts name and their surfaces

\begin{tabular}{cc}
\hline Parts Type & Surface $\left(\mathrm{mm}^{2}\right)$ \\
\hline A & $175 \times 40$ \\
B & $76 \times 76$ \\
C & $70 \times 50$ \\
D & $56 \times 47$ \\
E & $102 \times 19$ \\
F & $50 \times 35$ \\
\hline
\end{tabular}

Several cases can be studied, the parts are clustered into batches such as 500, 250, 125, 62, 31, 15 and 7 pieces for each one of the part types described in Table 2. This will enable us to study the impact of maximum utilization on the operations schedule.

\section{Methodology}

The analysis of the machine utilization in AM requires methods to carry out the study successfully. One of them is the implementation of suitable scenarios. This section presents a description of the main programming tool used in this work.

The utilization is defined as the ratio of the average output rate to maximum capacity. It is an important magnitude when several bundles of pieces must be printed and there is a set of printers, each one of them with a different build platform surface.

Several procedures could be chosen for solving these optimization problems. Therefore, it has been to implement a program by using the software package Python. The code is programmed to maximize the utilization of the machines for sets of batches. The inputs are the surface of the corresponding piece, the 3D printing build surfaces and the number of pieces per batch. The algorithm determines the set of printers that can print each batch with the feature that each printer harbours the number maximum of pieces per build platform. The program is running until the number of pieces per printer is greater than the rest of the pieces that remain to be printed, see Figure 2. If the maximum utilization is not reached i.e., there is a rest of the pieces that do not meet this condition, the program returns the percentage and the numbers of pieces that have not been printed.

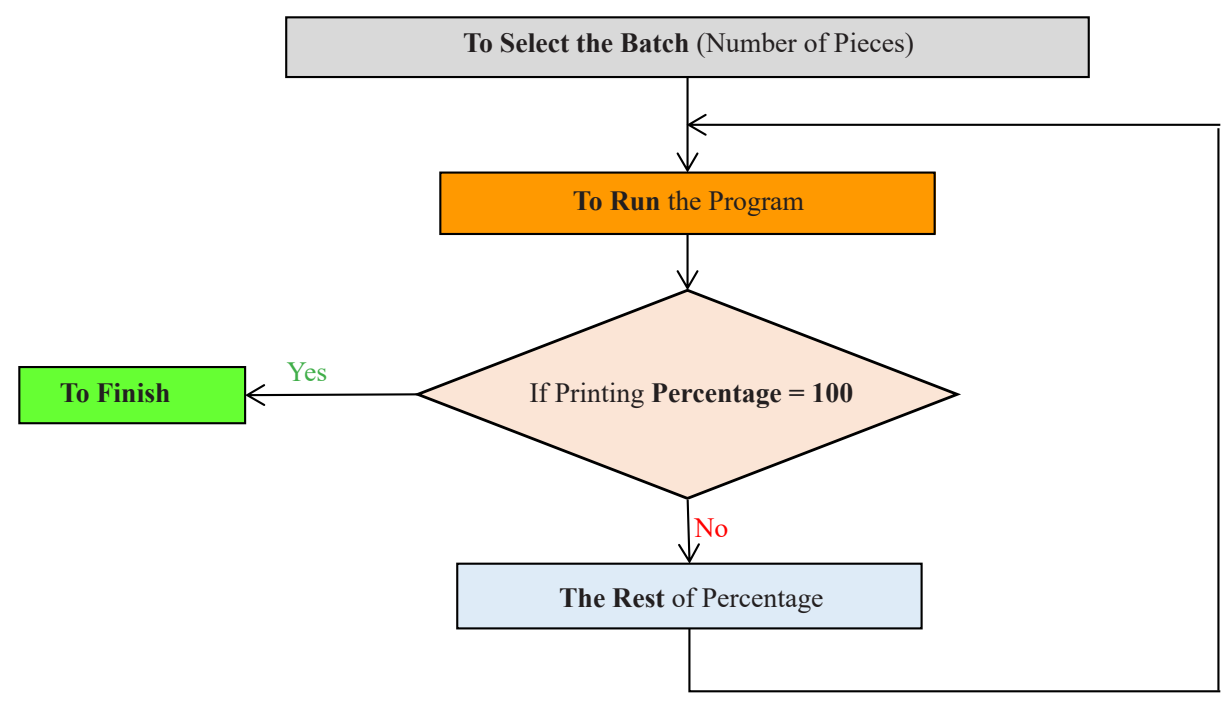

Figure 2. Flow chart of the program 
Regarding the maximum utilization, there are several scenarios for part selection. The first one is when an AM machine is full with the maximum number of parts that it can be printed. In this case, subtraction is done between the number of batch pieces and those assigned to the printer. This step will be doing until the number of parts can be allocated exactly to the printer. On the contrary, if the rest of them are less than the maximum number that the AM machines can print, this breaks the maximum utilization rule and the rest of the parts remain without printing. As it can be seen, there are two cases that must be highlighted, one if the batch can entirely be printed. Two, if there are a reduced number of parts then could not be printed.

The available areas on machines are updated every time that an assignation of parts is carried out to the printers' job. Hence, the parts assigned to the temporary job are removed from the list of available parts for the machines. Those parts that were not assigned become part of the list of available parts and they are updated after each assignment. This cycle continues until one of the previously described cases occurs.

As a result, the criteria to select the batches are determined by $3 \mathrm{D}$ printing build surfaces and the maximum utilization of them. The assignation is done by selecting the corresponding sets of pieces that can be printed that meet the above criteria. The weighting is given by the prevalence of the batches that can be entirely obtained by using the maximum utilization for the corresponding printers.

\section{Results}

This section presents the results of the program. Different cases are studied through combinations of a given number of parts and a number of printers. For each instance, a set of batches with different production areas are considered.

\subsection{AM machines and maximum utilization}

The first step to carry out the study is to regard some parts that will be stacked in batches. Some parameters are taken into account as constants, such as the printing speed and material used, among others. These ones could be considered variables, however; this goes beyond the aim of the work. In this way, the model is simplified or avoid the complex problem and unnecessary details. The names and the parts' surfaces are provided in Table 2.

Some aspects should be considered before the printing process such as the limited surface of the build platform and the number of pieces. For demonstrating purposes of the model, several batches are selected and the program is run. Table 1 provides an overview of the build platform surface for the simulation runs.

For these trials, the program uses the surfaces of the printing parts as well as the printers' surfaces in two dimensions i.e., the third dimension is not accounted for in the current study. The simulation is run using the program, which has been implemented using the software package Python. The aim is to measure the degree to which AM machines are being used, in order to know the ratio of the average output rate to maximum capacity. In addition, this allows obtaining information on the maximizing of the utilization of a process, which contributes to the competitive priority of cost (slack capacity).

The proposal of using the maximum utilization as an indicator that aids in the decision making in AM should be started by the study of the set of batches that can be entirely printed with the surfaces of the 3D printer full. If this is not possible, the rest of the pieces must be printed in an AM machine that will not be full.

Maximum utilization can be studied and an assignation can be also established between the numbers of printed parts per machine and the utility, see Figure 3. As can be seen, each figure shows the percentages of unprinted pieces versus the corresponding batches grouped by types of pieces. Figure 3 (A) shows the percentage of parts printed for type A grouped in different batches formed by 500, 250, 125, 62, 31, 15 and 7 pieces that can be completely printed with all the surfaces of the printer full in each case. Figure 3 (B) displays the percentage of parts printed for type B equally grouped in different batches i.e., 500, 250, 125, 62, 31, 15 and 7 pieces, in this case, only the batches of 31 and 15 can be completely printed with the surfaces of the printer full. The rest of the batches cannot be entirely printed; there is a rest of the pieces that must be printed without meeting the criteria of maximum utilization. Figure 3 (C) shows for pieces 
of type $\mathrm{C}$ that the batches of 500 and 62 cannot be completely printed and the rest of the pieces must be printed aside. Figure 3 (D) displays the type of piece $\mathrm{D}$ that can be printed for the most part except the batches of 500 and 7 pieces, respectively. As can be seen, in Figure 3 (E) the behaviour is opposite to the one shown in Figure 3 (A) i.e., the pieces of type E cannot be entirely printed in any case. Finally, Figure 3 (F) shows those three batches of pieces $F(250,31,7)$ that can be completely printed without any rest and four batches $(500,125,62$ and 15) that cannot be entirely printed, inhere, some parts must be printed without the surfaces of the printer full. The outcomes show that some batches cannot be successfully printed using the maximum utilization criterion.

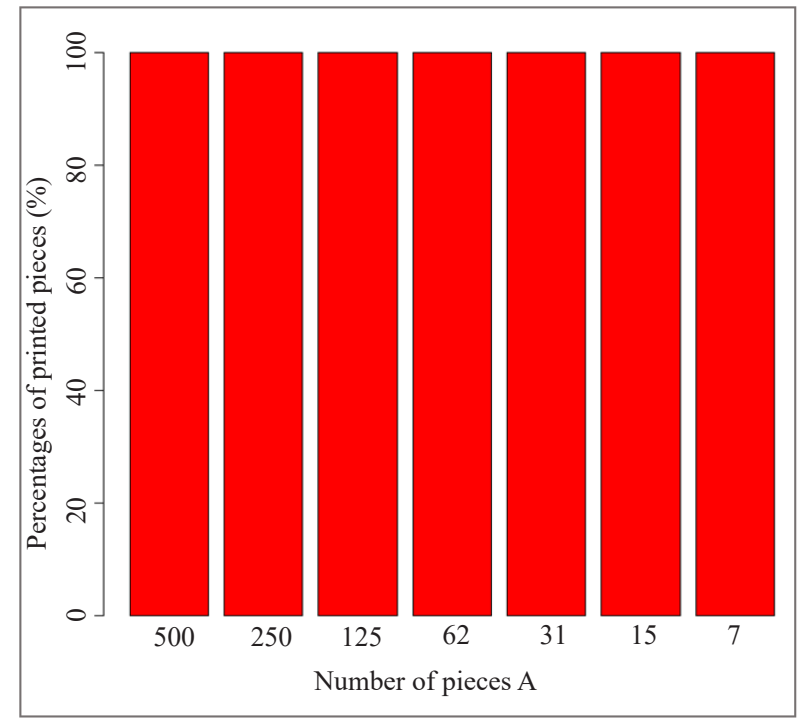

(A)

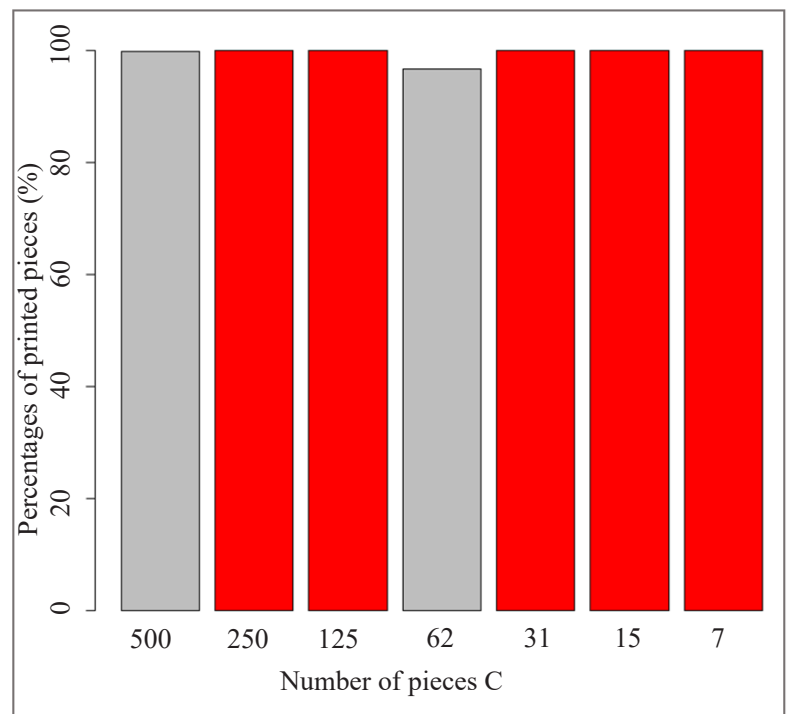

(C)

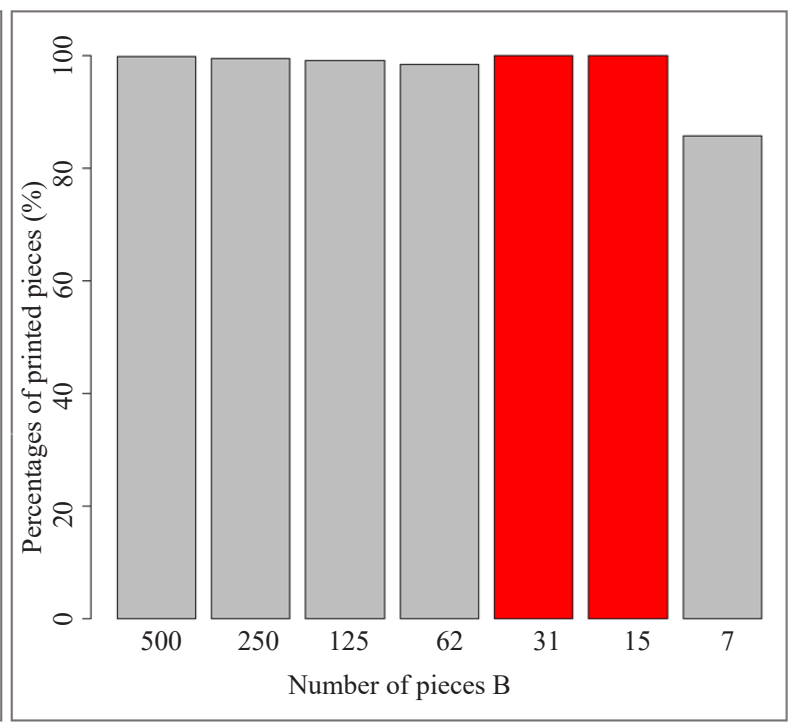

(B)

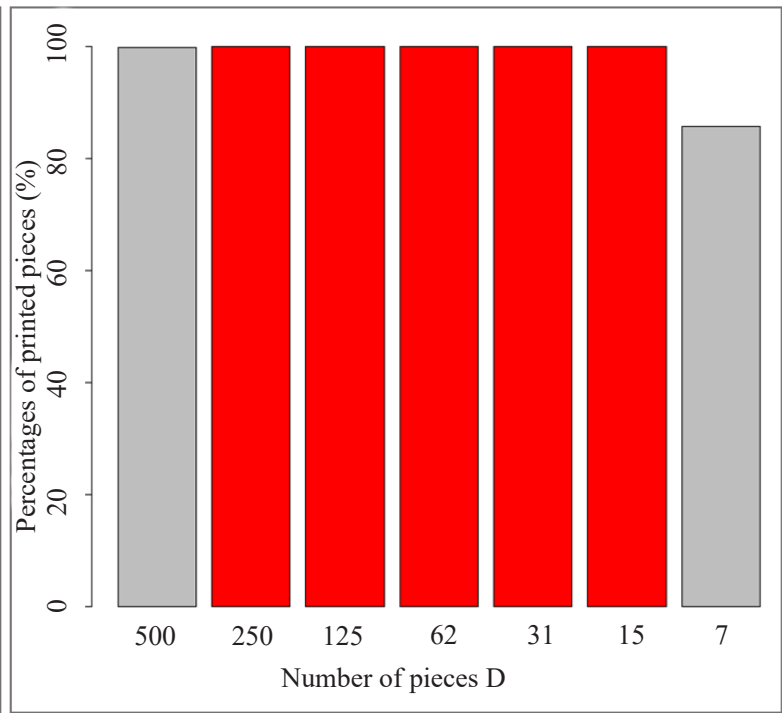

(D) 


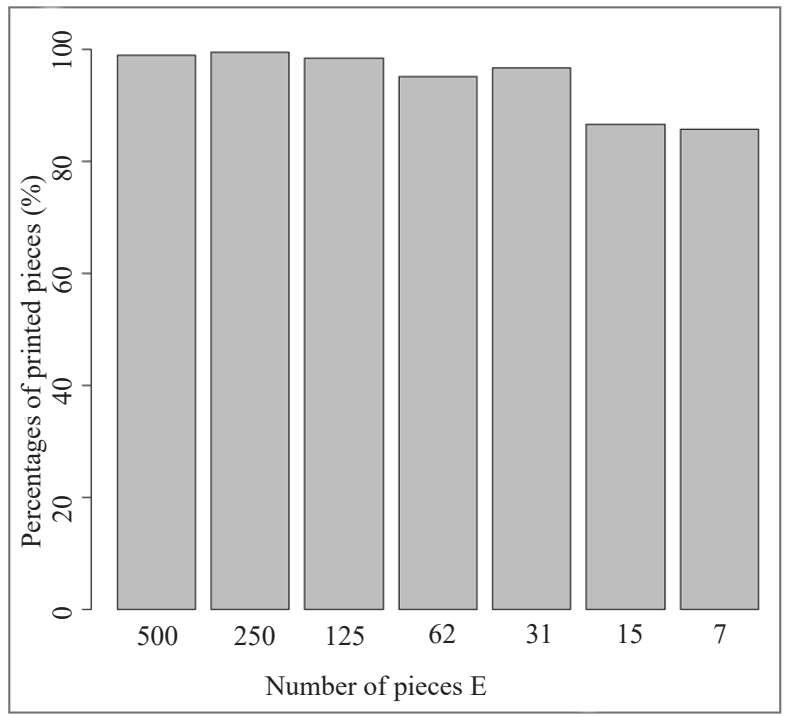

(E)

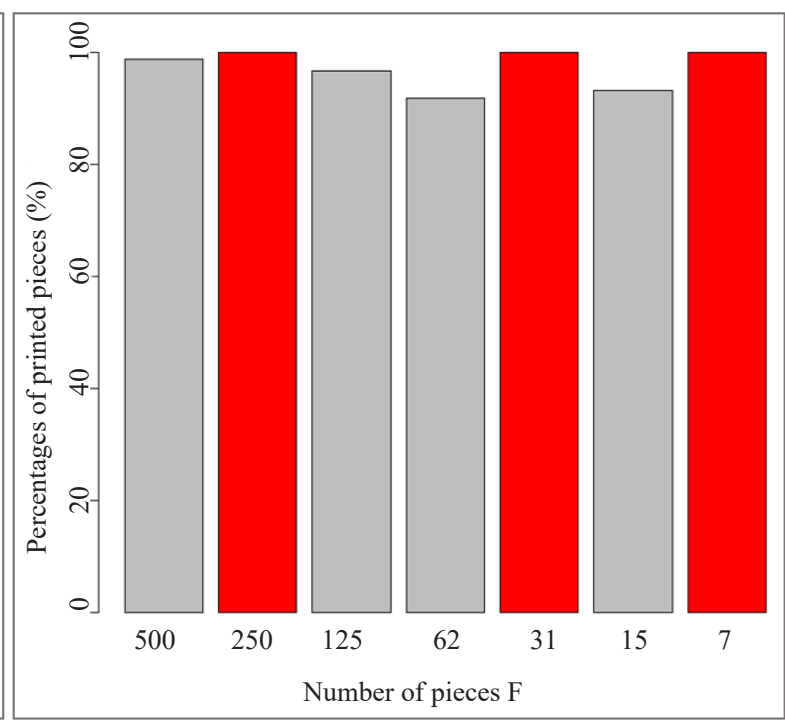

(F)

Figure 3. Figure (A) shows the percentages of printed pieces versus batches of 500, 250, 125, 62, 31, 15 and 7 of pieces type A. Figure (B), (C), (D), (E) and (F) display the percentages of printed pieces versus batches of 500, 250, 125, 62, 31, 15 and 7 of pieces type B, C, D, E and F, respectively. Red columns mean maximum utilization $(100 \%)$ and grey columns mean that maximum utilization has not been reached for the $3 \mathrm{D}$ printer surface for the set of ten printers available.

Once the maximum utilization has been studied when the type of pieces is considered, afterwards it is analyzed the role that the size of the batches on the scheduling. In these cases, the study is focused on the impact that the first interaction and its associated printer have on the programming. Figure 4 (A) shows the percentages of printed pieces for batches of 500 pieces for each type from A to F. As can be seen, pieces A display a percentage less than 30\% on the total number of pieces for the first interaction, and the pieces B show a percentage between $30 \%-40 \%$. The rest of the pieces have a percentage higher than 50\%. Figure 4 (B) shows as the percentages of printed pieces for the batches of 250 pieces of the types $\mathrm{E}$ and $\mathrm{F}$ are less than $30 \%$ the rest of the pieces have a percentage higher than 50\%. Figure 4 (C) displays the cases for batches formed by 125 pieces; pieces $\mathrm{C}$ have a percentage less than $30 \%$ and pieces $\mathrm{D}$ have a percentage between $30 \%-40 \%$, the rest of the batches show percentages higher than $40 \%$. Figure 4 (D) shows similar behaviour to Figure 1. Figures $4(\mathrm{E}),(\mathrm{F})$ and $(\mathrm{G})$ display behaviours higher than $50 \%$ for the percentage of pieces printed in the first round, there is no case the percentage is less than $40 \%$. 


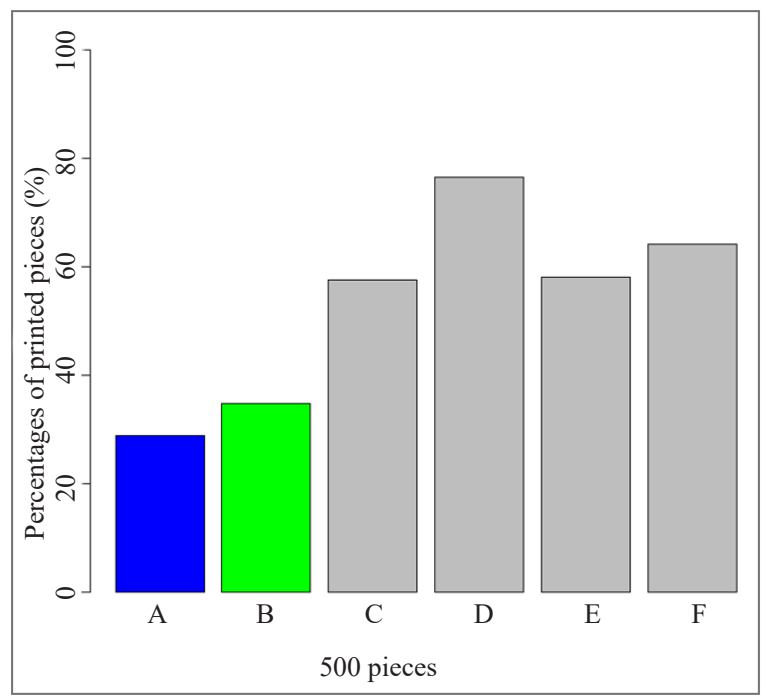

(A)

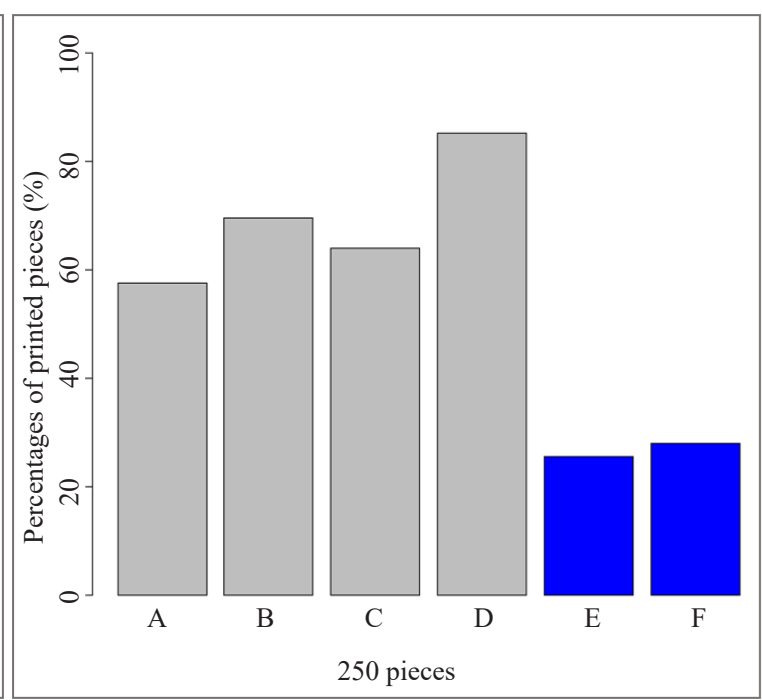

(B)

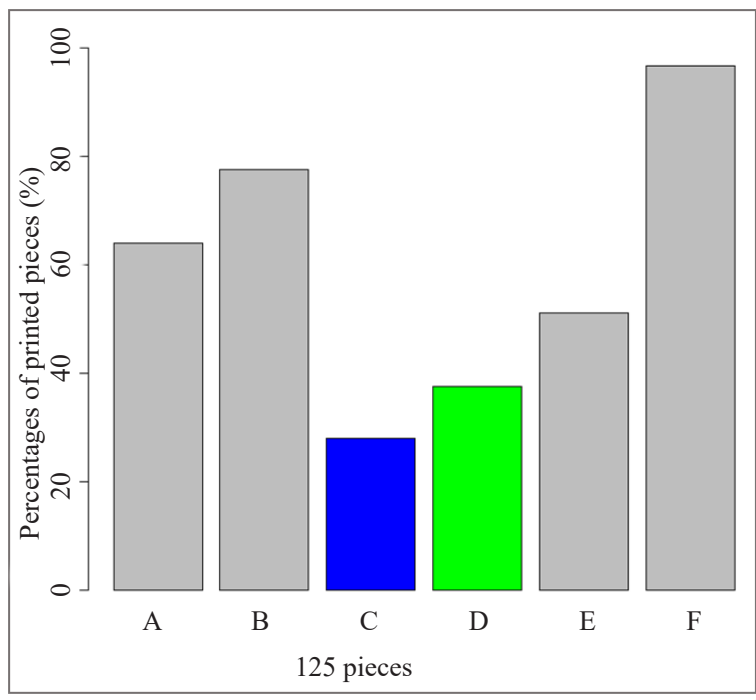

(C)

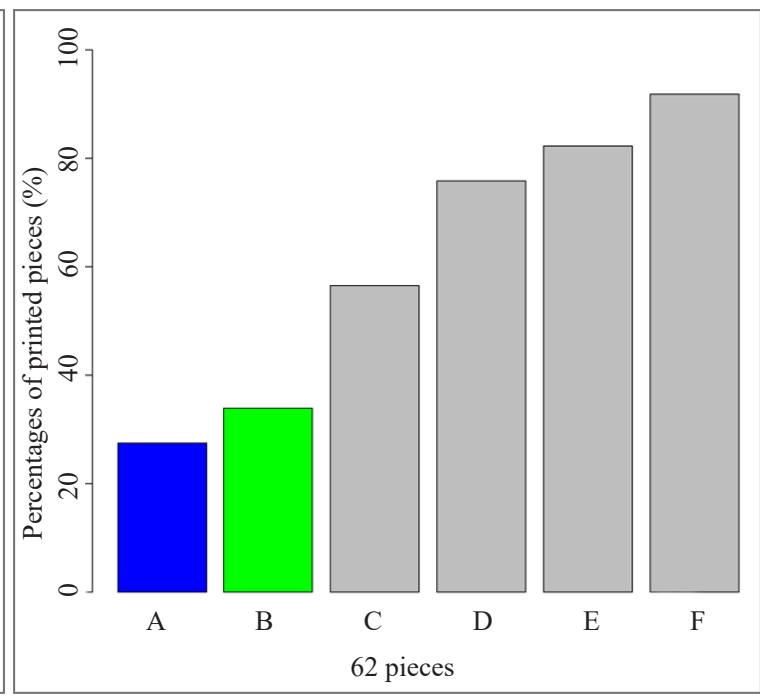

(D) 


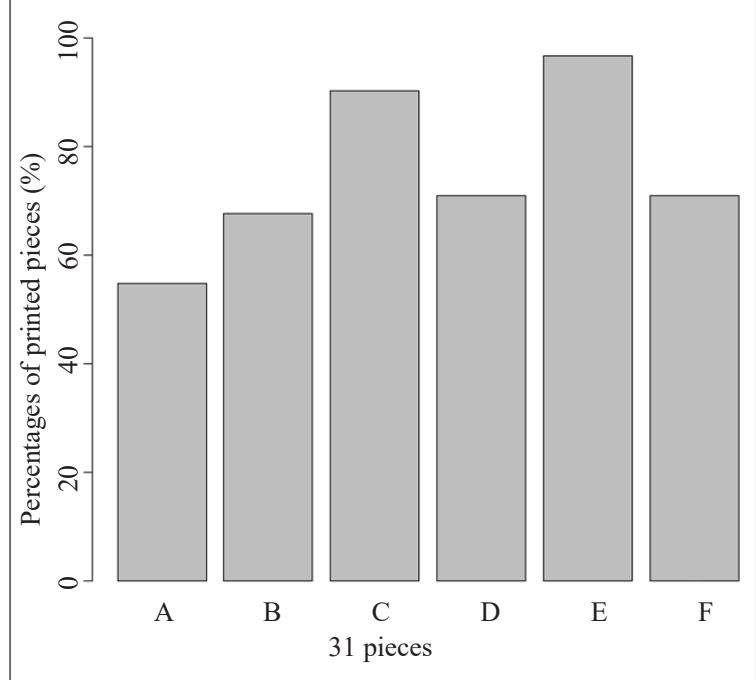

(E)

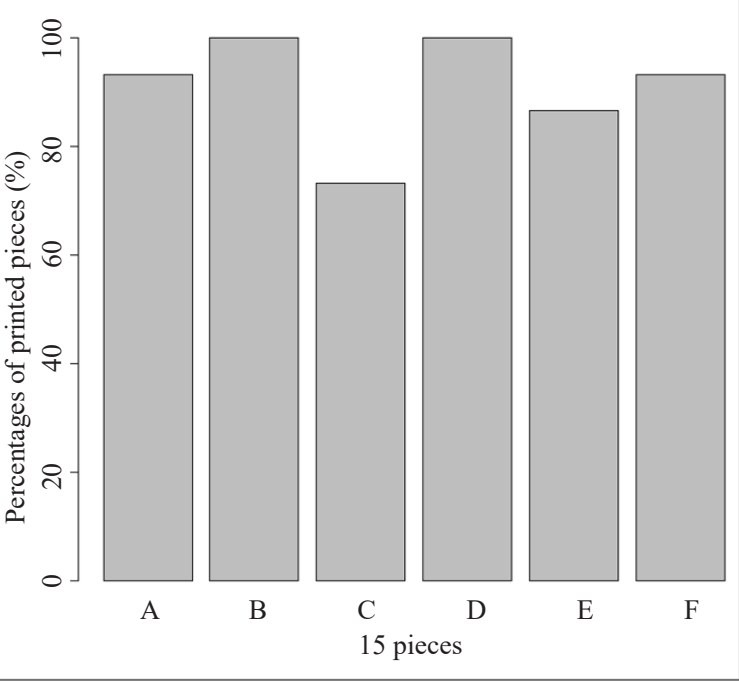

(F)

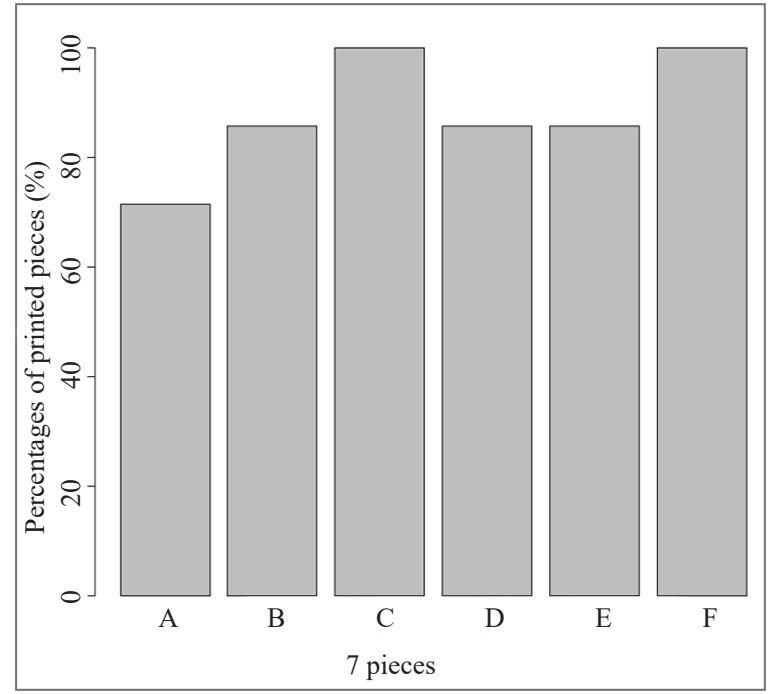

(G)

Figure 4. Figure (A) shows the percentages of printed pieces versus types of pieces A, B, C, D, E and F for batches of 500. Figure (B), (C), (D), (E) and $(\mathrm{F})$ display the percentages of printed pieces versus types of pieces A, B, C, D, E and F for batches of 250, 125, 62, 31 15, and 7, respectively. Blue columns mean maximum utilization of the first round less than $30 \%$. Green columns represent maximum utilization of the first round between $30 \%$ $40 \%$ and grey columns mean that maximum utilization is greater than $40 \%$.

Taking into account the outcomes presented in Figure 4, it is mandatory to study if the percentages obtained are less than $30 \%$ and those between $30 \%-40 \%$ have any impact on the scheduling. As a result, Figure 5 shows the reuse number versus the percentage of pieces printed. Figure $5(\mathrm{~A})$ displays that the reuse number is equal to three as in the first interaction the percentage is less than $30 \%$. This case means that for a batch of 500 pieces of type A the same printer will be used three times full. In addition, if the percentage is between $30 \%-40 \%$ for a batch of 500 pieces of type B, the printer that provided this percentage will be used twice. In another case, if the percentage is greater than $50 \%$ the reuse number is equal to one. Figure 5 (B) shows as the reuse number is three in two cases, for batches of 250 pieces of type $\mathrm{E}$ and $\mathrm{F}$. As the percentage is both of them is less than $30 \%$ the reuse number is three i.e., the printers that given these percentage will be used three times full. The rest of the batches for 250 pieces have a percentage of printed greater than 
$50 \%$ and the reuse numbers are equal to one. Figure $5(\mathrm{C})$ and (D) shows similar behaviour to Figure 5 (A), these cases are for batches 125 and 62 pieces. Figure $5(\mathrm{C})$ displays that pieces the types $\mathrm{C}$ and $\mathrm{D}$ have numbers of reuse three and two as expected considering the percentage of pieces printed in the first interaction, respectively. Figure 5 (D) shows analogous behaviour to the previous cases but for batches of pieces the types A and B. According to the percentages obtained in Figure $4(\mathrm{E}),(\mathrm{F})$ and $(\mathrm{F})$, the reuse numbers in Figure $5(\mathrm{E}),(\mathrm{F})$ and $(\mathrm{G})$ are one, as expected.

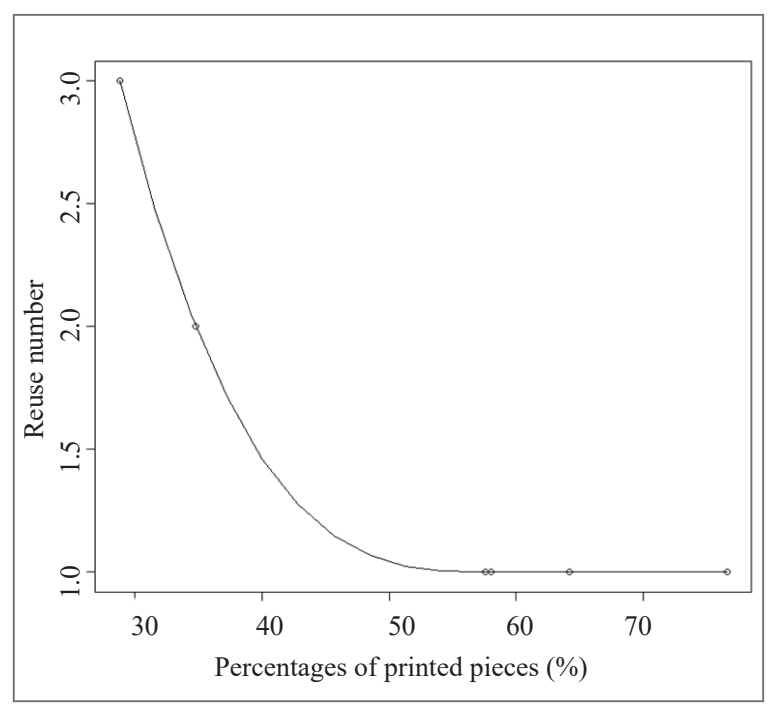

(A)
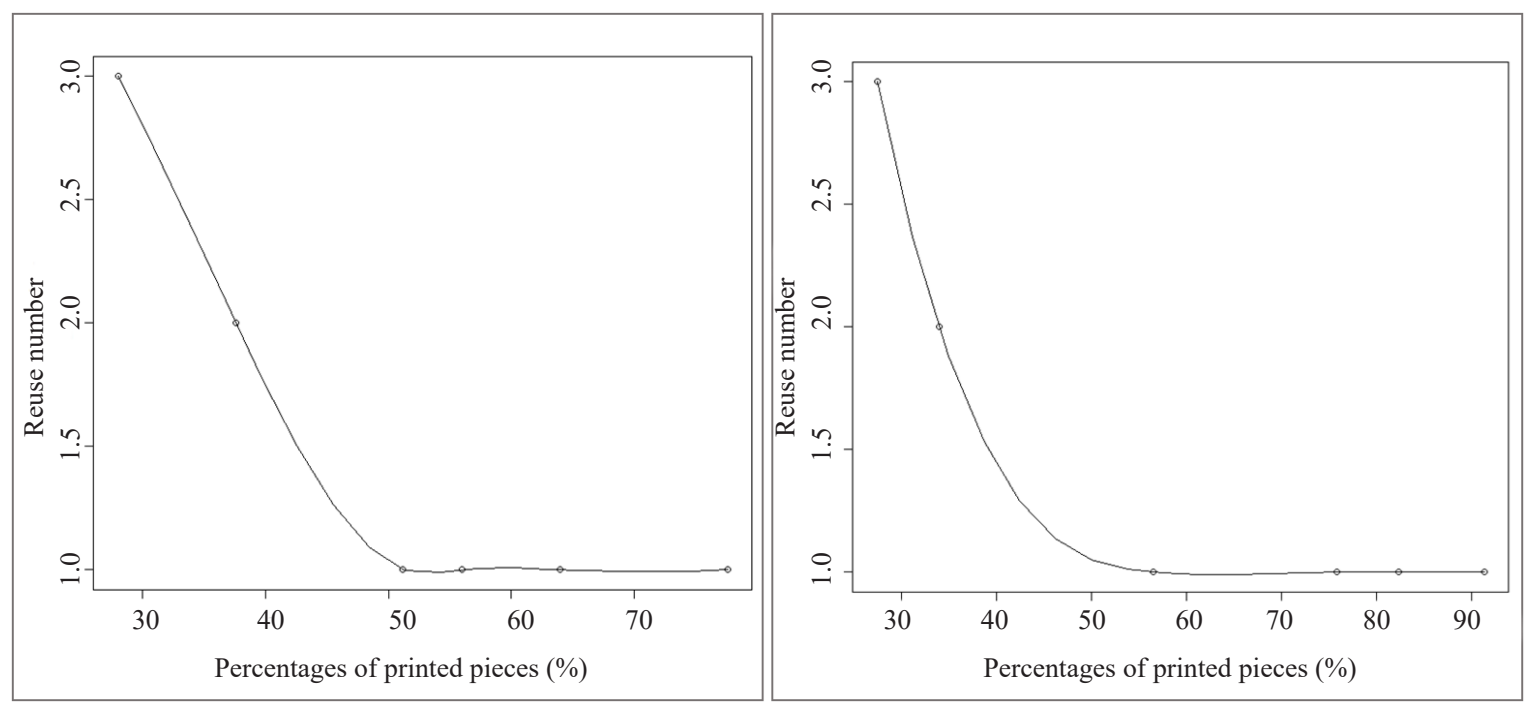

(D) 


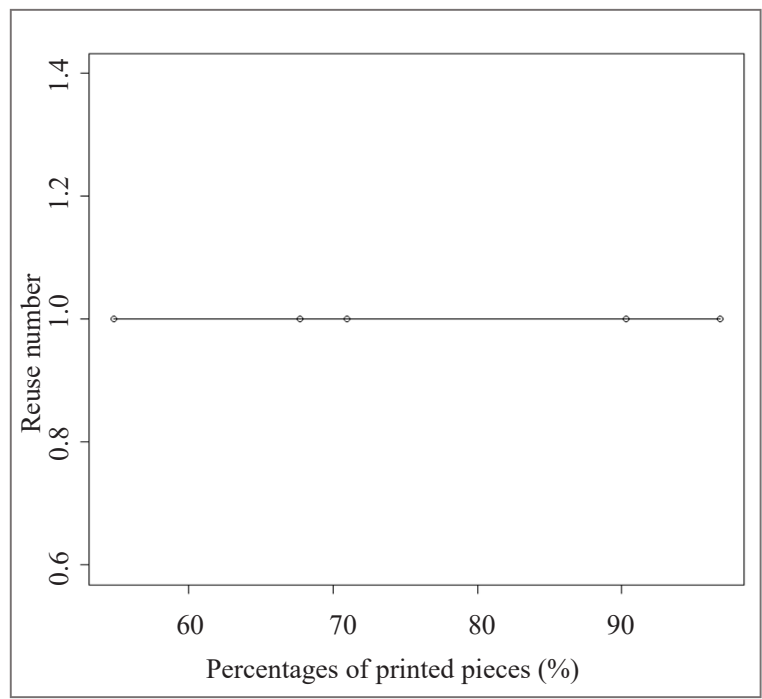

(E)

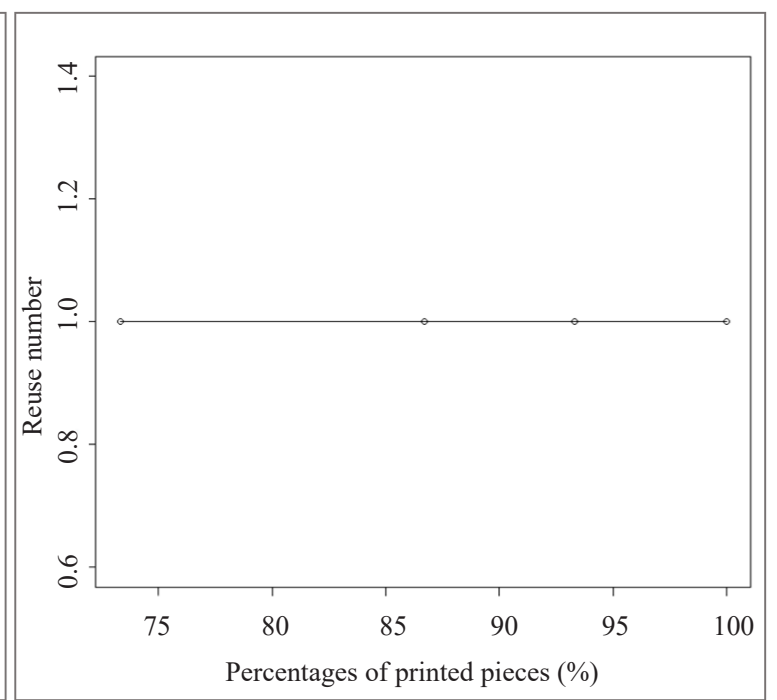

(F)

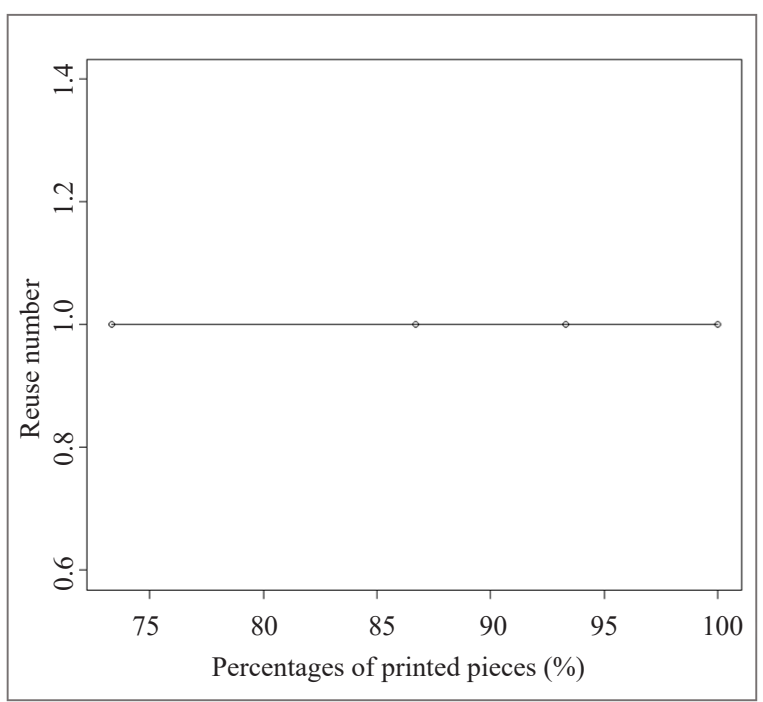

(G)

Figure 5. Each figure shows the corresponding reuse number versus the percentages of printed pieces of all the types (A, B, C, D, E and F). Figure (A) is for 500 pieces of all the types, Figure (B) 250 pieces of all the types, figure (C) 125 pieces of all the types, figure (D) 62 pieces of all the types, figure (E) 31 pieces of all the types, figure (F) 15 pieces of all the types and finally figure $(\mathrm{G}) 7$ pieces of all the types.

As can be seen, Figure 5 displays that as the percentage of the first interaction is less than $30 \%$ the corresponding printer is used three times and as the percentage is between $30 \%-40 \%$ the first $3 \mathrm{D}$ printer is used twice. On the other hand, if the percentage is greater than $40 \%$ the first AM machine is used only one time.

\subsection{Operations scheduling and maximum utilization}

Once the utilization has been studied the maximum utilization indicator has provided sufficient piece of information to determine the operation schedule.

There are two types of scenarios to operate scheduling such as machines in series and parallel. According to the results obtained in series can be used in form of the open shop to print the batches of pieces that are in blue and 
green colours in Figure 4 (A, B, C, D) at least with the first 3D printer selected to print the three or two jobs, if the corresponding percentage is less than $30 \%$ or it is between $30 \%-40 \%$, respectively. However, the rest of the figures of Figure 4 could be printed by parallel configuration in form of unrelated machines. As can be seen, in figures 4 and 5 as the number of pieces is less than a determined value, the reuse number does not show a magnitude greater than one.

On the other hand, if Figure 3 (A) is considered the series configuration in form of the open shop should be applied. On the contrary, Figure 3 (E) displays that parallel configuration should be used because there is no feasible to print the corresponding batches with maximum utilization and it is required to have some $3 \mathrm{D}$ printer in parallel to carry out the work successfully i.e., to have a printer ready to print the rest of the pieces that could not be printed by using the maximum utilization criterion. Regarding the majority of the outcomes, a hybrid system such as Flexible Flow Shop should be mostly used.

\section{Conclusions}

Nowadays, AM should cope with the assignation problem which it is being demanded by the market. As a consequence of this, a systematic method for studying the utilization of a set of batches and printers has been provided jointly. A review of the existing literature associated with AM and operation schedules have been done to shed light on these topics.

The main goal has been maximizing machines utilization, a program has been done and this has shown consistent performance due to the variability of the features in various optimization problems. A production line has been simulated to study the effect that the maximum utilization of the AM machines has on the operations schedule. The approach was set up based on the maximum utilization and it was programming using Python. Some cases were conducted to show the need of developing suitable AM planning and scheduling methods, to fulfil the technical and organizational requirements of $\mathrm{AM}$.

Once the maximum capacity is established per machine and the average output rate, the utilization can be maximized and this enables to determine the slack capacity. The assignation problem has been analyzed, which allowed us to calculate the set of printers that optimize the production considering the bundles. It has been found that if the percentage of the first round is less than $30 \%$ the AM machine is used three times. In addition, as the percentage is between $30 \%-40 \%$ the first 3D printer is used twice and if the percentage is greater than $40 \%$ the first $\mathrm{AD}$ machine is used only one time. These results have determined if the operation schedule can be established in series or parallel or Flexible Flow Shop.

This methodology can be run as a tool that provides appropriate information in the design of the 3D printing industry, being a decision instrument for companies that use AM technologies. The article has displayed a proper formalization of the outcomes which will enable us its implementation in further works.

\section{References}

[1] Weller C, Kleer R, Piller FT. Economic implications of 3D printing: Market structure models in light of additive manufacturing revisited. International Journal of Production Economics. 2015; 164: 43-56. Available from: doi: 10.1016/j.ijpe.2015.02.020.

[2] Dilberoglu UM, Gharehpapagh B, Yaman U, Dolen M. The role of additive manufacturing in the era of Industry 4.0. Procedia Manufacturing. 2017; 11: 545-554. Available from: doi: 10.1016/j.promfg.2017.07.148.

[3] Pour MA, Zanardini M, Bacchetti A, Zanoni S. Additive manufacturing impacts on productions and logistics systems. IFAC-PapersOnLine. 2016; 49(12): 1679-1684. Available from: doi: 10.1016/j.ifacol.2016.07.822.

[4] Demirel E, Özelkan EC, Lim C. Aggregate planning with flexibility requirements profile. International Journal of Production Economics. 2018; 202: 45-58. Available from: doi: 10.1016/j.ijpe.2018.05.001.

[5] Aloui A, Hadj-Hamou K. A heuristic approach for a scheduling problem in additive manufacturing under technological constraints. Computers \& Industrial Engineering. 2021; 154: 107115. Available from: doi: 10.1016/ j.cie.2021.107115.

[6] Li Q, Kucukkoc I, Zhang DZ. Production planning in additive manufacturing and 3D printing. Computers and 
Operations Research. 2017; 83: 157-172. Available from: doi: 10.1016/j.cor.2017.01.013.

[7] Chergui A, Hadj-Hamou K, Vignata F. Production scheduling and nesting in additive manufacturing. Computers \& Industrial Engineering. 2018; 126: 292-301. Available from: doi: 10.1016/j.cie.2018.09.048.

[8] Dvorak F, Micali M, Mathieu M. Planning and scheduling in additive manufacturing. Inteligencia Artificial. 2018; 21(62): 40-52. Available from: doi: 10.4114/intartif.vol21iss62pp40-52.

[9] Fera M, Fruggiero F, Lambiase A, Macchiaroli R. State of the art of additive manufacturing: Review for tolerances, mechanical resistance and production costs. Cogent Engineering. 2016; 3(1): 1261503. Available from: doi: 10.1080/23311916.2016.1261503.

[10] Fera M, Fruggiero F, Costabile G, Lambiase A, Pham D. A new mixed production cost allocation model for additive manufacturing (MiProCAMAM). Int $J$ Adv Manuf Technol. 2017; 92: 4275-4291. Available from: doi: 10.1007/s00170-017-0492-x.

[11] Costabile G, Fera M, Fruggiero F, Lambiase A, Pham D. Cost models of additive manufacturing: A literature review. International Journal of Industrial Engineering Computations. 2017; 8: 263-283. Available from: doi: 10.5267/j.ijiec.2016.9.001.

[12] Stittgen T, Schleifenbaum JH. Simulation of utilization for LPBF manufacturing systems. Production Engineering. 2021; 15: 45-56. Available from: doi: 10.1007/s11740-020-00998-1.

[13] Fera M, Macchiaroli R, Fruggiero F, Lambiase A. A modified tabu search algorithm for the single-machine scheduling problem using additive manufacturing technology. International Journal of Industrial Engineering Computations. 2020; 11: 401-414. Available from: doi: 10.5267/j.ijiec.2020.1.001.

[14] Czerniachowska K, Zywicki K, Wichniarek R. A method to improve planning of product placement on a printing sheet. Management and Production Engineering Review. 2021; 12(1): 119-128. Available from: doi: 10.24425/ mper.2021.136877.

[15] Araújo LJP, Özcan E, Atkin J, Baumers M, Tuck C, Hague R. Toward better build volume packing in additive manufacturing: classification of existing problems and benchmarks. In 26th Annual International Solid Freeform Fabrication Symposium-an Additive Manufacturing Conference. 2015; 401-410. Available from: https:// nottingham-repository.worktribe.com/output/759177. [Accessed August 2015].

[16] Lodi A, Martello S, Monaci M. Two-dimensional packing problems: A survey. European Journal of Operational Research. 2002; 141(2): 241-252. Available from: doi: 10.1016/S0377-2217(02)00123-6.

[17] Renner G, Ekart A. Genetic algorithms in computer aided design. Computer-Aided Design. 2003; 35(8): 709-726. Available from: doi: 10.1016/S0010-4485(03)00003-4.

[18] Vaissier B, Pernot JP, Chougrani L, Véron P. Genetic-algorithm based framework for lattice support structure optimization in additive manufacturing. Computer-Aided Design. 2019; 110: 11-23. Available from: https://sam. ensam.eu/handle/10985/16940. [Accessed October 2019]

[19] Takakuwa S. The use of simulation in activity-based costing for flexible manufacturing systems. In Proceedings of the 29th conference on Winter simulation: 1997; 12: 793-800. Available from: doi: 10.1145/268437.268657.

[20] Castillo-Rivera S, De Antón J, Del Olmo R, Pajares J, López-Paredes A. Genetic algorithms for the scheduling in additive manufacturing. International Journal of Production Management and Engineering. 2020; 8(2): 59-63. Available from: doi: 10.4995/ijpme.2020.12173.

[21] Chung SH, Chan FTS, Chan HK. A modified genetic algorithm approach for scheduling of perfect maintenance in distributed production scheduling. Engineering Applications of Artificial Intelligence. 2009; 22(7): 1005-1014. Available from: doi: 10.1016/j.engappai.2008.11.004.

[22] Mohamed OA, Masood SH, Bhowmik JL. Optimization of fused deposition modeling process parameters: a review of current research and future prospects. Advances in Manufacturing. 2015; 3(1): 42-53. Available from: doi: 10.1007/s40436-014-0097-7.

[23] Dey A, Yodo N. A systematic survey of FDM process parameter optimization and their influence on part characteristics. Journal of Manufacturing and Materials Processing. 2019; 3(3): 64. Available from: doi: 10.3390/ jmmp3030064.

[24] Popescu D, Zapciu A, Amza C, Baciu F, Marinescu R. FDM process parameters influence over the mechanical properties of polymer specimens: A review. Polymer Testing. 2018; 69: 157-166. Available from: doi: 10.1016/ j.polymertesting.2018.05.020. 